\section{Eukaryotic Conditional Expression System}

BioTechniques 27:106-110(July 1999)

\begin{abstract}
Existing conditional expression systems can be classified in two major categories that are based either on the induction or on the de-repression of transcription. The system described here combines both mechanisms, since a unique transcription factor can be shifted from a repression to a stimulation activity by simply changing its ligand. The resulting advantage of this system is the complete absence of basal expression before active induction. The principle of this method is based on the unexpected ability of the chimeric protein containing the DNA-binding domain of the yeast Gal4 transcription factor fused to the $\mathrm{COOH}$ half of the estradiol receptor (GalER), to act as a repressor when bound to the drug $4 \mathrm{OH}$ tamoxifen, in the context of a previously described optimized Gal4-responsive promoter. The efficacy of this system has been assessed in transient expression assays using the chloramphenicol acetyl transferase (CAT), and in situ, through the activity of a Gal4 responsive $\beta$-galactosidase gene.
\end{abstract}

\section{INTRODUCTION}

Since no protein is completely inert when ectopically expressed, controlling transgene expression is a major challenge in biotechnology. The tight regulation of transgenes is a prerequisite for producing proteins of medical or economical interest that display some toxic or cytostatic properties for cells and organisms. In a more fundamental point of view, conditional expression systems will become a tool of choice, complementary to gene inactivation, to gain insight into the function of the unknown genes provided by systematic sequencing strategies. Two symmetrical approaches have been used to develop effector-driven expression systems: $(i)$ the induction method, using ligand-dependent transcription machineries and (ii) the de-repression method, using repressors that can be shifted in a liganddependent manner into conformations unable to bind DNA. These latter tools more often derive from prokaryotic gene repressors and their corresponding operator DNA-binding sites, as the tetracyclin system (4) and the lactose operator-repressor system (10).

The major limitation of the available inducible expression systems is the presence of a basal expression level in absence of active induction. This problem is likely a result of the spontaneous activity of the minimal promoters used in transgene constructions and by virtually any DNA fragment, due to the presence of cryptic binding sites for known or unknown transcription factors. This drawback is particularly obvious in the induction systems using cis-elements recognized by endogenous transcription factors like heatshock elements, nuclear receptor target sites or heavy-metal responsive promoters.

The present method is based on the use of a chimeric transcription factor containing the DNA-binding domain from the yeast Gal4 transcription factor (Gal-DBD), which is known to have no equivalent in higher eukaryotes, thus avoiding undesirable interferences with endogenous transcription regulators. In the present case, Gal-DBD is fused to the ligand-dependent transcription activation domain from the human estradiol receptor (ER)-LBD. The resulting chimeric transcription factor, named GalER, has long been shown to be capable of enhancing the activity of a synthetic Gal4-responsive promoter after the addition of estrogen (9). The chimeric GalER factor presents two favorable features: $(i)$ the presence of the activation domain from the carboxyterminal end of the ER (AF2), which is well defined and clearly estradiol-dependent and, in turn, (ii) the absence of the $\mathrm{NH}_{2}$-terminal domain of the ER (AF1), that displays some ligand-independent activity, which is unfavorable for the present purpose. However, the interest of such an estrogen-inducible system was greatly hindered by: (i) the presence of endogenous estrogens in body fluids or in the serums incorporated into cell culture media and (ii) by the spontaneous basal activity of Gal4-responsive promoters. To circumvent this problem, a synthetic Gal4-reponsive promoter has been constructed, with extremely low basal activity (2). This optimized construct contains four palindromic Gal4-binding sites, linked to TATA and CCAAT boxes. However, we observed that this promoter still presents a significant level of activity in the absence of induction. Here, we present an additional improvement of this GalER system, allowing in the same time, the repression of the basal expression activity and a stimulation of greater amplitude.

\section{MATERIALS AND METHODS}

\section{Materials}

4-hydroxy-tamoxifen (4OH-tamoxifen) and estradiol were from Sigma (L'isle d'Abeau Chesnes, St. Quentin Fallavier, France). The reporter plasmid GalREnlslacZ was created by inserting a blunted fragment encoding the nuclear $\beta$-galactosidase ( $\beta$-gal) (1) into the EcoRV site of GC vector, which carries the Gal4-responsive promoter (2). The Gal-chloramphenicol acetyl transferase (CAT) reporter plasmid is the pCAT-4 construct described previously (2) and was kindly provided by Dr. Meinrad Busslinger (Research Institute of Molecular Pathology, Vienna, Austria). The GalER expression plasmid, described by Webster et al. (9), was kindly provided by Prof. Pierre Chambon (IGBMC Strasbourg, France).

\section{Cell Culture}

CHO-CH3S cells were maintained at $37^{\circ} \mathrm{C}$ in $5 \% \mathrm{CO}_{2}$ atmosphere, in $\mathrm{F} 12-$ Dulbecco's modified Eagle medium (1:1) supplemented with $10 \%$ dextran charcoal-treated fetal bovine serum (FBS) (Life Technologies, Gaithersburg, MD, USA). Phenol Red ${ }^{\circledR}$ was omitted because of its weak estrogenic activity. Medium was added with antibiotic/antimycotic solution composed of penicillin $(500 \mathrm{U} / \mu \mathrm{L})$, streptomycin $(500$ $\mu \mathrm{g} / \mu \mathrm{L})$ and amphotericin $(1.25 \mu \mathrm{g} / \mu \mathrm{L})$.

\section{Transfections}

Transfections were performed using a modified calcium phosphate precipitate method (2). Cells were seeded in 6-well tissue culture plates at the 
density of $10^{5}$ cells/well one day before transfection. One hour before adding calcium phosphate/DNA co-precipitate, cells were provided with fresh medium either with or without $4 \mathrm{OH}$ - tamoxifen $(1 \mu \mathrm{M})$. Twenty-four hours later, precipitate was removed by phosphate-buffered saline (PBS) washing and replaced by fresh medium containing either 4OH-tamoxifen or estradiol $(1 \mu \mathrm{M}$ each). Cells were then grown for transient expression during $24 \mathrm{~h}$ and then submitted to either protein extraction for CAT assays or in situ LacZ detection.

\section{CAT Assays}

Cells transfected with Gal-CAT and GalER, along with cytomegalovirus (CMV) $\beta$-gal as an internal control of transfection, were harvested after $48 \mathrm{~h}$ transient expression by scraping in chilled PBS containing 5\% glycerol and centrifuged. Cell pellets were resuspended in $130 \mu \mathrm{L}$ of Tris- $\mathrm{HCl}, \mathrm{pH} 7.8$,

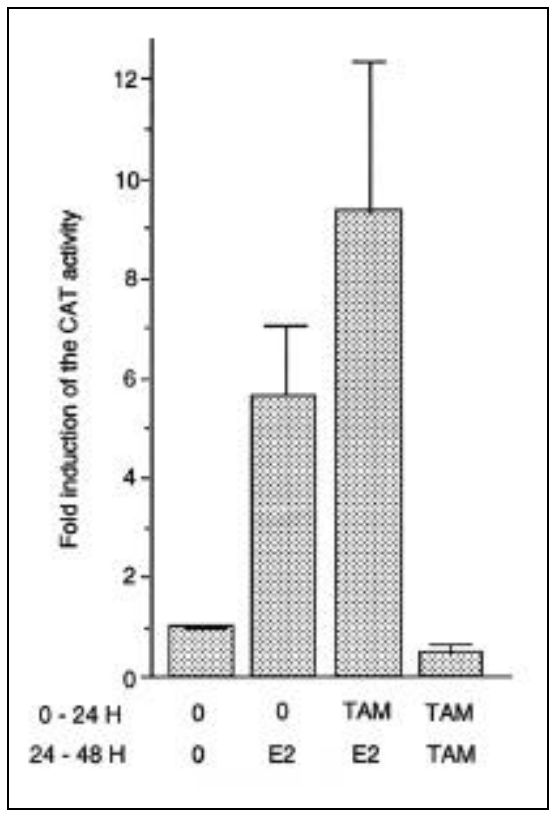

Figure 1. CHO-CH3S cells $\left(1.5 \times 10^{5}\right.$ cells/ well) were co-transfected with the Gal-CAT reporter plasmid and a GalER expression vector, along with $C M V \beta$-gal as an internal control of transfection. Cultures were treated with or without estradiol (E2) or 4OH-tamoxifen (TAM) during $48 \mathrm{~h}$, or successively with TAM and then E2, as indicated under the histogram. Cells lysates were then tested for CAT activity as described in Materials and Methods. Results are expressed as -fold induction of the CAT activity relative to cells treated with the solvent alone (ethanol) (means $\pm \mathrm{SD} ; n=3$ ). containing $0.5 \%$ Triton ${ }^{\circledR} \mathrm{X}-100$ and then frozen and thawed twice to improve lysis. Cellular debris were then eliminated by centrifugation, and $98-\mu \mathrm{L}$ aliquots of the supernatants were submitted to a 10 -min incubation at $65^{\circ} \mathrm{C}$ to remove potential CAT inhibitors. Enzymatic activity was determinated using the following protocol: $98 \mu \mathrm{L}$ of cell extracts were put in presence of $20 \mu \mathrm{L}$ chloramphenicol, $5 \mathrm{mM}$ in Tris- $\mathrm{HCl}$ (250 mM), pH 7.8, 0.5 $\mu \mathrm{L}$ acetyl-CoA (both from Boehringer Mannheim GmbH, Mannheim, Germany) (4.54 $\mu \mathrm{M}), \quad 0.4 \mu \mathrm{L} \quad\left[{ }^{3} \mathrm{H}\right]$ acetyl-CoA $(200$ $\mu \mathrm{Ci} / \mu \mathrm{M}$; ICN Biomedicals, Orsay, France) and $19.1 \mu \mathrm{L} \mathrm{HCl}(75 \mu \mathrm{M})$. After a 3 -h incubation at $37^{\circ} \mathrm{C}$, reactions were stopped by adding $1 \mathrm{~mL}$ of $5 \mathrm{M}$ urea. $\beta$-gal activities were determinated using the uncolored substrate of this enzyme, namely the ortho-nitro-phenyl- $\beta$ D-galactopyranoside (ONPG; Boehringer Mannheim $\mathrm{GmbH}$ ) as follows: $30 \mu \mathrm{L}$ of lysate were incubated with $330 \mu \mathrm{g}$ ONPG $(1.1 \mu \mathrm{g} / \mu \mathrm{L})$ in sodium phosphate buffer ( $\mathrm{pH} 7.5$ ). Reaction products were then assayed by spectrophotometric measurement at $450 \mathrm{~nm}$. The relative CAT activities were expressed as CAT/ $\beta$-gal ratios. These ratios were compared to determine the fold activations of the system.

\section{In Situ Detection of $\mathbf{L a c Z}$-Positive Cells}

Cells were initially fixed by a 15-min incubation in $1 \%$ glutaraldehyde solution at room temperature. After washing in PBS, they were then incubated at $37^{\circ} \mathrm{C}$ for $90 \mathrm{~min}$ in a coloration solution containing 5-bromo-4chloro-3-indyl- $\beta$-D-galactoside (X-gal; Life Technologies) as a colorless substrate of $\beta$-gal. After removing the coloration solution, cells were covered with $70 \%$ glycerol and observed by light microscopy.

\section{RESULTS}

The synthetic Gal4-responsive promoter with extremely low basal activity (2) was used to direct the expression of a modified $L a c Z$ coding region containing the nuclear localization signal from the simian virus 40 (SV40) large T anti- 


\section{Short Technical Reports}

gen (GalRE-nlsLacZ). The nuclear concentration of $L a c Z$ gene product further facilitates the detection of cells expressing LacZ at low level. The Gal4-responsive promoter used in this construction was also to be activated by the chimeric transcription factor GalER in presence of estradiol and to display a low spontaneous activity (2). However, when introduced into Chinese hamster ovary $(\mathrm{CHO})$ cells, we observed a significant activity even in absence of GalER, as demonstrated for instance, by in situ LacZ staining after introduction of the GalRE-nlsLacZ plasmid (Figure 2A, column 1). Such a background expression is likely to prohibit the use of this system for transgenes that can affect cell viability. When attempting to lower this basal activity, we found that in presence of GalER, 4OHtamoxifen, a low-priced compound known for its ability to act as an antiestrogen by preventing estradiol-dependent transcription stimulation by ER (8), can significantly repress the level of background transcription. In CAT assays, done in presence of GalER, the sustained presence of $4 \mathrm{OH}$-tamoxifen in the culture medium decreases, by more than half, the basal CAT activity (Figure 1, columns 1 and 3). In addition, preincubation of cells with $4 \mathrm{OH}-$ tamoxifen before estrogen addition, leads to an unexpected enhancement of the stimulating action by estrogen. Combined with its lessening effect on the basal expression, this enhancing activity allows a dramatic increase of the amplitude of induction by estrogen. Indeed, estrogen addition at $24 \mathrm{~h}$ leads to a 5.6-fold activation of the Gal4-responsive promoter (Figure 1, columns 1 and 2), while a 4OH-tamoxifen pretreatment from $0-24 \mathrm{~h}$ leads to a 19.4-fold induction (Figure 1, compare columns 3 and 4). In fact, this effect of $4 \mathrm{OH}$-tamoxifen, which is very useful in the context of this system, could have been expected as a result of the previous observation that estradiol stimulation of the ER can be enhanced by previous addition of an anti-estrogen (5).

We have also tested the validity of the system through the in situ $\beta$-gal detection assay. This alternative method was also used to determine if GalER is involved in mediating the effects of estradiol and 40H-tamoxifen. As shown in Figure 2, the induction by estradiol of the number of blue cells is estradiol receptor-dependent, considering the 4-fold increase of LacZ-positive cells induced by the presence of GalER (Figure 2A, compare columns 1 and 3). One must notice, however, that the LacZ staining is more intense upon stimulation by estradiol (data not shown), which might reflect a certain stimulating effect of estradiol. By itself, 4OH-tamoxifen seems to have an estrogen receptor-independent stimulating effect on transgene expression (Figure 2A, column 2). In presence of GalER, 4OH-tamoxifen appears capable of

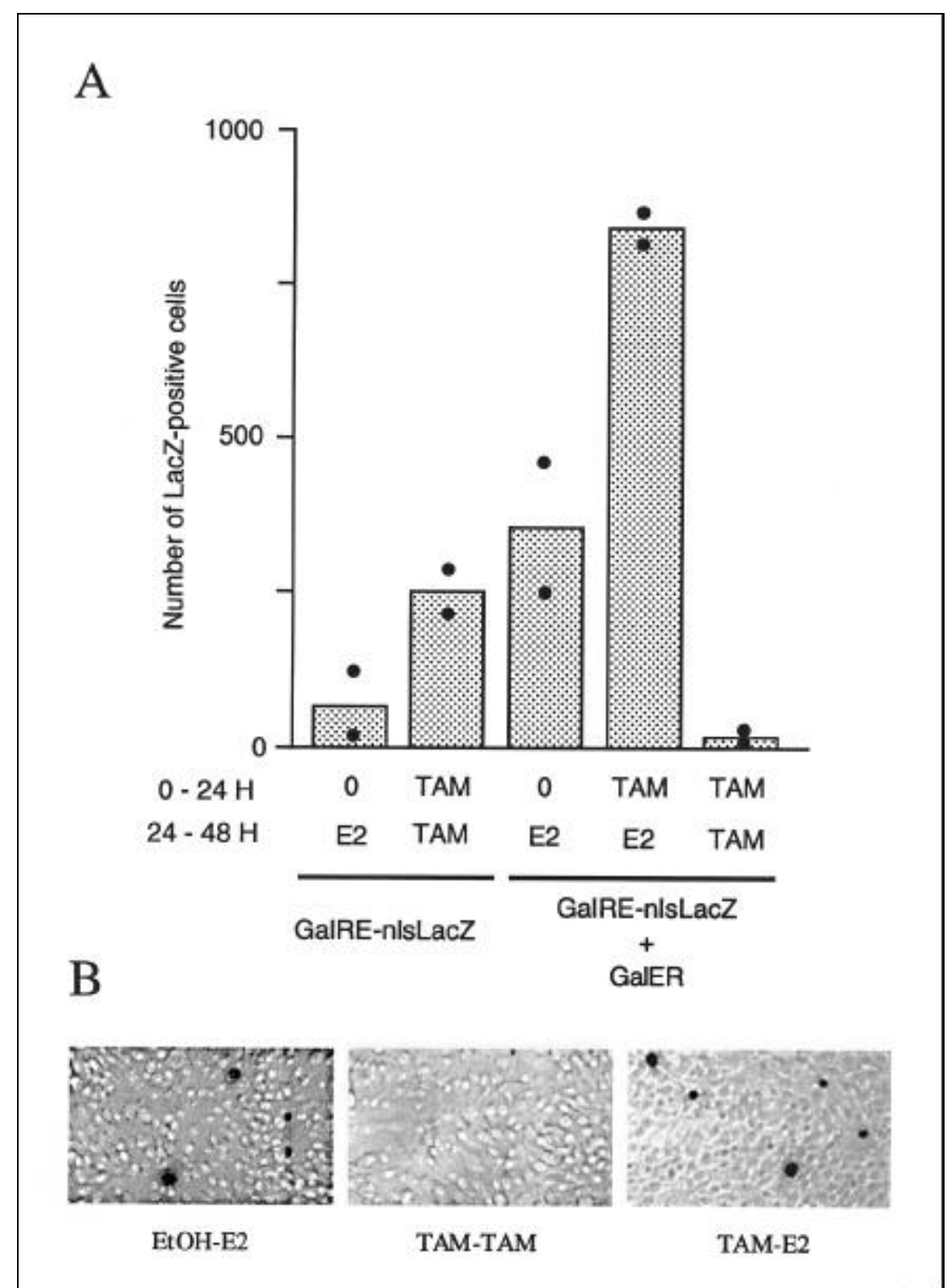

Figure 2. Effects of successive treatments by estradiol and/or 4OH-tamoxifen on the GalER-mediated activity of GalRE-nlsLacZ. (A) Number of LacZ-positive cells obtained after transfection with the GalRE-nlsLacZ reporter plasmid alone or co-transfected with an expression vector of GalER by the calcium phosphate method. 4OH-tamoxifen (TAM) was or was not added together with the calcium phospate DNA precipitate. Twenty-four hours later, the precipitate was washed and replaced by a fresh medium containing $1 \mu \mathrm{M}$ TAM or estradiol (E2). This figure is obtained from two independent experiments (dots). (B) Representative regions of dishes co-transfected with GalRE-nlsLacZ and GalER and LacZ-stained $48 \mathrm{~h}$ after transfection. Cells were treated with solvent from $0-24 \mathrm{~h}$ and then E2 from 24-48 h (left panel), with TAM from 0-48 h (middle) or with TAM from 0-24 h and then with E2 (right). 
strongly inhibiting the basal expression of the system (Figure 2A, column 5). This 4OH-tamoxifen-induced repression by GalER is powerful because very few LacZ-positive cells are detected after 4OH-tamoxifen pretreatment. For only 2 blue cells observed in one experiment in the presence of $4 \mathrm{OH}$-tamoxifen, more than 800 were observed in an identical dish after replacement of $4 \mathrm{OH}-$ tamoxifen by estrogen. Moreover, considering that GalER and the LacZ genes are carried by two different plasmids transfected in an equimolar ratio, one cannot exclude that these few cells are precisely those containing only the GalRE-nlsLacZ reporter plasmid. This drastic repressive effect of $4 \mathrm{OH}$-tamoxifen cannot result from a mere negative effect of this compound on transfection efficiency or on cell viability, since it is clearly dependent on the presence of GalER (Figure 2A, compare columns 2 and 5). In addition, the effect of 4OH-tamoxifen is also fully reversible because it does not forbid the action of a subsequent estradiol treatment, as demonstrated by the high inducibility by estradiol after 4OH-tamoxifen withdrawal. Indeed, as previously observed in CAT assays, the stimulating action of estradiol appears of higher amplitude if cells containing both GalER and GalRE-nlsLacZ are pretreated with $4 \mathrm{OH}$-tamoxifen. 4OH-tamoxifen pretreatment enhances the subsequent stimulating effect of estradiol, leading to a 2.4-fold increase of the number of LacZ-positive cells (Figure 2A, compare columns 3 and 4). The conditions used for Figure 2A, column 4, appear optimal for using GalER. Thus, as observed through the two techniques, CAT assays and X-gal staining, the use of tamoxifen greatly improves the use of the GalER/Gal4-RE system at two levels: $(i)$ by reducing the background expression and (ii) by enhancing the induction step.

\section{DISCUSSION}

We show that the conditional expression system, based on the Gal4-responsive promoter constructed by Braselmann et al. (2), can be strikingly improved by using the drug $4 \mathrm{OH}$-tamoxifen. Fortuitously, we found that in addition to the enhanced inducibility by estradiol, 4OH-tamoxifen strongly decreases the basal activity of the Gal4responsive promoter. This effect is clear in CAT assays and particularly obvious when using the in situ $\beta$-gal staining. The apparently more stringent regulation revealed by the latter method is probably inherent in the requirement for a threshold basal concentration of $\beta$-gal to be detectable. By itself, the repressive activity of 4OH-tamoxifen mediated by GalER, might appear surprising because contrary to other nuclear receptors, as for example the unliganded thyroid hormone (11), reports on a repressive activity of the estradiol receptor are very few. Interestingly, it

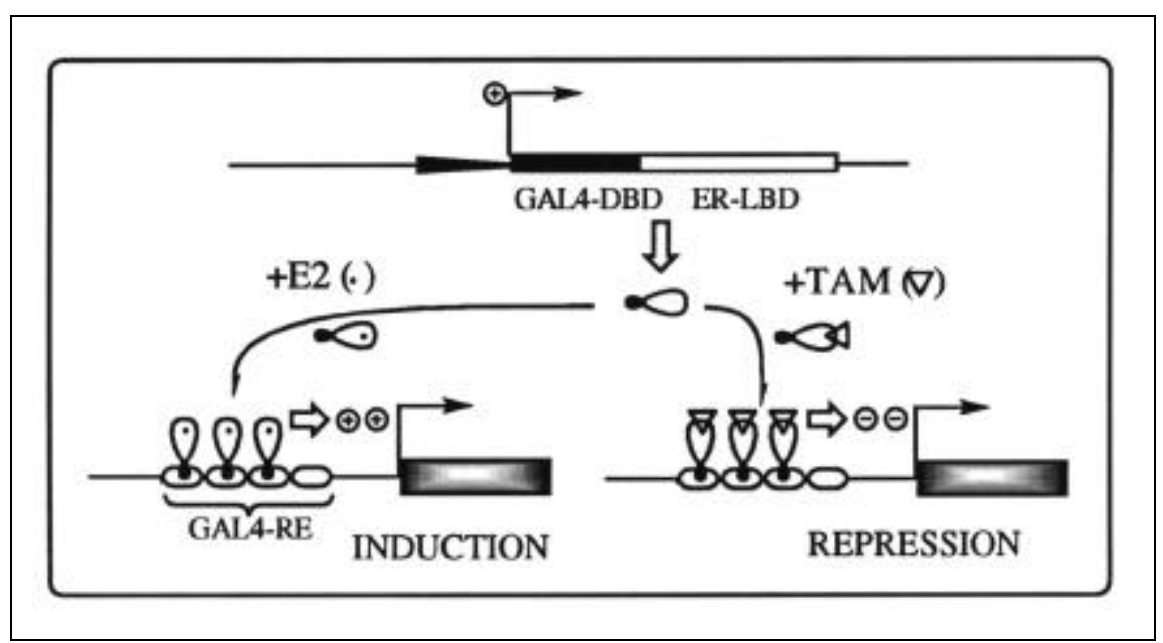

Figure 3. Schematic representation of the GalER-tamoxifen system. The same chimeric transcription factor, GalER, has the dual capacity of stimulating or inhibiting the transcription activity of the Gal4-responsive promoter, accordingly, as the ligand added is estradiol or tamoxifen, respectively. 
has been described that the AF2 domain of the estrogen receptor, present in GalER, can acquire a repressive activity through interaction with C/EBP (7). This finding is of particular interest considering that the Gal4-responsive promoter used in this system does contain a typical C/EBP target CCAAT box. It has also recently been shown that tamoxifen can promote the binding of co-repressors to the ER (6). Elucidating the molecular mechanism underlying the 4OH-tamoxifen-induced repression would be of fundamental interest. In the context of this work, this feature makes it possible to shift from a tight repression to a tight induction of the expression by using a single transcription factor (see Figure 3 for a synthetical scheme of the system). While only one out of the two activities of induction and repression is exploited at the same time in the other conditional expression systems, they are reunited in the present one, as a result of the dual activity of $4 \mathrm{OH}$-tamoxifen. Taken together, the absence of Gal4-RE-binding factors and $\mathrm{Gal} 4$ consensus recognition sites in higher eukaryotes, the lack of cellular toxicity of 4OH-tamoxifen and of estradiol and the strong repressive activity of 4OH-tamoxifen-bound GalER make this system particularly useful for finely regulating transgene expression. As an ultimate test, we plan to use this system for controlling the expression of toxic transgenes. In conclusion, the improved system presented here allows the reconciliation of most requirements for an ideal inducible system; specifically, a strong activity upon induction and, more importantly, a low activity before induction.

\section{REFERENCES}

1.Bonnerot, C., D. Rocancourt, P. Briand, G. Grimber and J.F. Nicolas. 1987. A betagalactosidase hybrid protein targeted to nuclei as a marker for developmental studies. Proc. Natl. Acad. Sci. USA 84:6795-6799.

2.Braselmann, S., P. Graninger and M. Busslinger. 1993. A selective transcriptional induction system for mammalian cells based on Gal4-estrogen receptor fusion proteins. Proc. Natl. Acad. Sci. USA 90:1657-1661.

3.Chen, C. and H. Okayama. 1987. High-efficiency transformation of mammalian cells by plasmid DNA. Mol. Cell Biol. 7:2745-2752.

4.Gossen, M. and H. Bujard. 1992. Tight control of gene expression in mammalian cells by tetracycline-responsive promoters. Proc. Natl. Acad. Sci. USA 89:5547-5551.

5.Lascombe, I., P. Mougin, C. Vuillermoz, G.L. Adessi and M. Jouvenot. 1995. Gene transfer into subcultured endometrial cells using lipofection. BioTechniques 20:88-91.

6.Lavinsky, R.M., K. Jepsen, T. Heinzel, J. Torchia, T.M. Mullen, R. Schiff, A. Del-Rio, M. Ricote et al. 1998. Diverse signaling pathways modulate nuclear receptor recruitment of N-CoR and SMRT complexes. Proc. Natl. Acad. Sci. USA 95:2920-2925.

7.Stein, B. and M.X. Xang. 1995. Repression of the interleukin- 6 promoter by estrogen receptor is mediated by NF- $\kappa \mathrm{B}$ and $\mathrm{C} / \mathrm{EBP} \beta$. Mol. Cell Biol. 15:4971-4979.

8.Sutherland, R.L., L.C. Murphy, M. San Foo, M.D. Green, A.M. Whybourne and Z.S. Krozowski. 1980. High-affinity anti-estrogen binding site distinct from the estrogen receptor. Nature 288:273-275.

9.Webster, N.J., S. Green, J.R. Jin and P. Chambon. 1988. The hormone-binding domains of the estrogen and glucocorticoid receptors contain an inducible transcription activation function. Cell 54:199-207.

10.Wu, J.D., H.C. Hsueh, W.T. Huang, H.S. Liu, H.W. Leung, Y.R. Ho, M.T. Lin and M.D. Lai. 1997. The inducible lactose operator-repressor system is functional in the whole animal. DNA Cell Biol. 16:17-22.

11.Zhang, J., I. Zamir and M.A. Lazar. 1997. Differential recognition of liganded and unliganded thyroid hormone receptor by retinoid $\mathrm{X}$ receptor regulates transcriptional repression. Mol. Cell Biol. 17:6887-6897.

This work was funded by Education Nationale (DRED), Centre National de la Recherche Scientifique (CNRS) and supported by the Association pour la Recherche contre le Cancer (ARC). Address correspondence to Prof. Denis Michel, UPRESA 6026 CNRS-Université de Rennes 1, Campus de Beaulieu, 35042 Rennes Cedex, France. Internet: denis.michel @univ-rennesl.fr

Received 8 September 1998; accepted 5 April 1999.

T. Sourisseau, Y. Le Dréan, G. Salbert, F. Flamant ${ }^{1}$ and D. Michel

CNRS

Université de Rennes 1

Rennes

${ }^{1}$ CNRS-ENS de Lyon

Lyon, France
Purification of Proteins

Fused to Either the

Amino or Carboxy

Terminus of the

Mycobacterium xenopi

Gyrase A Intein

BioTechniques 27:110-120 (July 1999)

\section{ABSTRACT}

The Mycobacterium xenopi gyrase $A$ mini-intein has been engineered to yield a controllable $N$-terminal or $C$-terminal, single-splice-junction autocleavage element. When combined with an affinity tag, these modified mini-inteins can be used to purify target proteins after a single combined chromatography/cleavage step. Cleavage at the intein $N$ terminus was induced with thiol reagents, while cleavage at the intein $C$ terminus was induced by a temperature shift to $16^{\circ}-25^{\circ} \mathrm{C}$. Different preferences for the residue immediately preceding the intein were observed during thiol-induced, $N$-terminal splice-junction cleavage of the $\mathrm{M}$. xenopi gyrase A mini-intein vs. the Saccharomyces cerevisiae vacuolar ATPase, subunit A (VMA) intein present in the IMPACT ${ }^{\mathrm{TM}}$ purification system. Furthermore, the M. xenopi gyrase A mini-intein $C$ terminal autocleavage vector allows isolation of polypeptides with $N$-terminal cysteine residues that are active in the Intein Mediated Protein Ligation method of protein semisynthesis.

\section{INTRODUCTION}

Many single-step systems are available to over-express and purify proteins fused to affinity tags, such as the Escherichia coli maltose binding protein (MBP), the Schistosoma japonicum glutathione S-transferase (GST), Histag, chitin binding domain (CBD), antibody epitope tags etc. (7). The subsequent proteolytic cleavage of the fusion protein to separate the target protein from the affinity tag can be problematic in these systems (7). Proteases are often inefficient, especially when the cleavage site is buried within the fusion 